Research Article

\title{
Dynamic Behaviors of a Two-Degree-of-Freedom Impact Oscillator with Two-Sided Constraints
}

\author{
Songtao Li $\mathbb{D}$, Qunhong Li $\mathbb{D}$, and Zhongchuan Meng $\mathbb{D}$ \\ College of Mathematics and Information Science, Guangxi University, Nanning 530004, China \\ Correspondence should be addressed to Qunhong Li; liqh@gxu.edu.cn
}

Received 25 August 2020; Revised 28 February 2021; Accepted 22 March 2021; Published 1 April 2021

Academic Editor: Zengshun Chen

Copyright (C) 2021 Songtao Li et al. This is an open access article distributed under the Creative Commons Attribution License, which permits unrestricted use, distribution, and reproduction in any medium, provided the original work is properly cited.

\begin{abstract}
The dynamic model of a vibroimpact system subjected to harmonic excitation with symmetric elastic constraints is investigated with analytical and numerical methods. The codimension-one bifurcation diagrams with respect to frequency of the excitation are obtained by means of the continuation technique, and the different types of bifurcations are detected, such as grazing bifurcation, saddle-node bifurcation, and period-doubling bifurcation, which predicts the complexity of the system considered. Based on the grazing phenomenon obtained, the zero-time-discontinuity mapping is extended from the single constraint system presented in the literature to the two-sided elastic constraint system discussed in this paper. The Poincare mapping of double grazing periodic motion is derived, and this compound mapping is applied to obtain the existence conditions of codimension-two grazing bifurcation point of the system. According to the deduced theoretical result, the grazing curve and the codimension-two grazing bifurcation points are validated by numerical simulation. Finally, various types of periodic-impact motions near the codimensiontwo grazing bifurcation point are illustrated through the unfolding diagram and phase diagrams.
\end{abstract}

\section{Introduction}

In mechanical engineering, there exist the vibroimpact phenomena widely, and systems interacting via impact have been extensively studied in recent years. Over the past years, scholars have mainly dedicated themselves to study the bifurcation phenomenon in smooth systems. Recently, a lot of work has gone into investigating nonsmooth bifurcations [1-8] of dynamical systems. The focus of investigations has gradually begun to change from a unilateral constraint system [9-11] to a multiconstraint system [12-17]. The impact oscillators can be divided into rigid or elastic impact oscillators according to the hardness of constraint. Shaw and Holmes [18] studied the motions of a system with a single piece rigid stop by using a one-dimensional mapping. Jiang and Wiercigroch [19] developed the concept of discontinuity geometry of rigid impact oscillators into the elastic impact oscillators, and the geometry analysis methods are applied to study the mechanisms of grazing bifurcations of system with unilateral soft constraint. Ing et al. [20] applied the methods of theoretical analyses and simulation to study different bifurcation scenarios for an impact oscillator with one-sided amplitude constraint. Du et al. [21] studied the intermittent chaos control method of a symmetrical collision system with the two-degree-of-freedom elastic double-impact system. The controlling idea of the Hopf bifurcation was applied to the system to provide a new control method for the chaos control of such system. Gritli et al. [22, 23] considered a state-feedback controller and applied the Linear Matrix Inequality (LMI) approach to control the motion of the system subject to norm-bounded parametric uncertainties. Shen et al. $[24,25]$ proposed a discrete feedback controller to suppress grazing-induced motion. Gritli and Belghith [26-29] considered the dynamic behavior of the model under the OGY-based state-feedback control, and the bifurcation phenomena were carried out via the bifurcation diagrams. Chávez et al. [30] studied a single-parameter and two-parameter bifurcations of the system that consists of two identical Duffing oscillators interacting via soft impact with the aid of COCO.

As a special bifurcation of nonsmooth systems, the grazing bifurcation is a qualitative transition between impact 
and nonimpact motion [31-46]. Nordmark [31,32] studied the dynamic behavior near the grazing trajectory and a nonlinear mapping is obtained. The normal form mapping for grazing bifurcation in an n-dimensional impact system is obtained in [35]. The existence condition and stability of the grazing periodic orbit are derived in a two degree-of-freedom vibroimpact system with one-sided constraint in [36]. Yin et al. [37-42] analyzed and measured the degenerate grazing bifurcations of the impact systems. The dynamic behavior of the grazing bifurcation is investigated by using the Poincare composite mapping technology, and the conditions of the degenerate grazing bifurcation are found. And the relationship between the observed bifurcations and degenerate grazing point is presented by a two-parameter continuation. Kowalczyk et al. [43] made a simple classification of codimension-two bifurcations of nonsmooth systems. Dankowicz and Zhao [44] deduced specific formulae for the local map on the vicinity of the codimensiontwo points and presented the bifurcation behavior by numerical simulations. The codimension-two grazing bifurcations in single-degree-of-freedom impact oscillators are studied and the dynamic response near the bifurcation points is presented in [45]. Xu et al. [46] investigated the codimension-two grazing bifurcation in n-degree-of-freedom impact oscillator with bilateral constraints by using a classical approach of discontinuity mappings.

Based on previous studies, we discuss the bifurcation behavior of the system with a two-sided elastic constraint by using the path-following method. And then, abundant and complex bifurcation behaviors are exhibited, such as grazing bifurcation, saddle-node bifurcation, fold bifurcation, period-doubling bifurcation. Much attention has been paid to analyze codimension-two bifurcation of a system with rigid impact, little work has been studied on the analysis of such bifurcation in the soft impact system. And then, on the basis of discontinuity mappings of the rigid vibroimpact system, the zero-time-discontinuity mappings $\mathbf{Z D M}_{1}, \mathbf{Z D M}_{2}$ of the elastic constraint vibroimpact system are deduced. And the compound mapping $\mathbf{P}$ is applied to obtain the existence conditions and specific mathematical expressions of codimension-two grazing bifurcation point.

This paper is discussed as follows: in section 2 we introduce a dynamic model of a two-degree-of-freedom vibroimpact system with two-sided soft constraints. The codimension-1 bifurcation analysis of the system is presented in section 3 . In section 4 , we derive a Poincare mapping near the grazing bifurcation by using the zerodiscontinuity-mapping method; the numerical simulations according to the deduced theoretical result are presented. In Section 5, the conclusions are given.

\section{Physical Model and Equations of Motion}

2.1. Physical Model. A physical model for the two-degree-offreedom impact oscillator with masses $M_{1}$ and $M_{2}$ is described in Figure 1. The masses $M_{1}$ and $M_{2}$ are connected

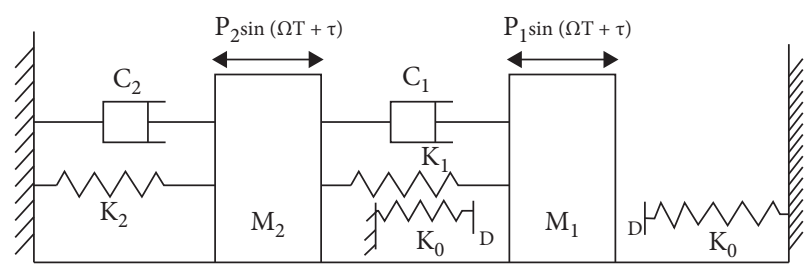

FIgURE 1: The model of the two-degree-of-freedom vibroimpact system with symmetrical constraints.

via the linear springs and linear viscous dampers, and the mass $M_{1}$ is limited by the symmetrical elastic constraint corresponding to two discontinuity surfaces $\mathbf{D}_{1}$ and $\mathbf{D}_{2}$. The excitations on the masses $M_{1}$ and $M_{2}$ are harmonic forces with amplitudes $P_{1}, P_{2}$, respectively. The excitation frequency and the phase are the same for the masses, where the frequency is taken as the controlling parameter in the following: when the displacement $X_{1}$ of the oscillator is $(D($ or $-D))$, it will collide with the right (or left) elastic constraint. Damping in this model is considered as proportional damping of the Rayleigh type, which implies $C_{1} / K_{1}=C_{2} / K_{2}$.

2.2. Differential Equation of Motion. The equation of motion of the physical model in Figure 1 can be expressed as follows:

$$
\begin{gathered}
M_{1} \ddot{X}_{1}+K_{1}\left(X_{1}-X_{2}\right)+C_{1}\left(\dot{X}_{1}-\dot{X}_{2}\right)+F_{c} \\
=P_{1} \sin (\Omega T+\tau), \\
M_{2} \ddot{X}_{2}+K_{2} X_{2}+C_{2} \dot{X}_{2}+K_{1}\left(X_{2}-X_{1}\right)+C_{1}\left(\dot{X}_{2}-\dot{X}_{1}\right) \\
=P_{2} \sin (\Omega T+\tau), \\
F_{c}= \begin{cases}K_{0}\left(X_{1}+D\right), & X_{1} \leq-D, \\
0, & -D<X_{1}<D, \\
K_{0}\left(X_{1}-D\right), & X_{1} \geq D .\end{cases}
\end{gathered}
$$

Introduce the following dimensionless variables and time:

$$
\begin{gathered}
\mu_{m}=\frac{M_{2}}{M_{1}}, \mu_{k 1}=\frac{K_{2}}{K_{1}}, \mu_{c}=\frac{C_{2}}{C_{1}}, \mu_{k 0}=\frac{K_{0}}{K_{1}}, f_{2}=\frac{P_{2}}{P_{0}}, t=T \sqrt{\frac{K_{1}}{M_{1}}}, \\
\omega=\Omega \sqrt{\frac{K_{1}}{M_{1}}}, \zeta=\frac{C_{1}}{2 \sqrt{K_{1} M_{1}}}, d=\frac{D K_{1}}{P_{0}}, x_{i}=\frac{X_{i} K_{1}}{P_{0}}, \quad i=1,2 .
\end{gathered}
$$

Systems (1) and (2) can be expressed in a normalized form as

$$
\mathbf{M} \ddot{x}+2 \mathbf{Z} \dot{x}+\mathbf{K x}+\mathbf{f}_{c}=\mathbf{F},
$$

in which 


$$
\begin{aligned}
& \mathbf{M}=\left(\begin{array}{ll}
1 & 0 \\
0 & \mu_{m}
\end{array}\right), \\
& \mathbf{K}=\left(\begin{array}{cc}
1 & -1 \\
-1 & 1+\mu_{k 1}
\end{array}\right), \\
& \mathbf{Z}=\zeta \mathbf{K}=\left(\begin{array}{cc}
\zeta & -\zeta \\
-\zeta \zeta \times\left(1+\mu_{c}\right)
\end{array}\right), \\
& \mathbf{F}=\left(\begin{array}{c}
1-f_{2} \\
f_{2}
\end{array}\right), \\
& \mathbf{f}_{c}=\left\{\begin{array}{cc}
\left(\mu_{k 0}\left(x_{1}+d\right), 0\right)^{T}, & x_{1} \leq-d, \\
(0,0)^{T}, & -d<x_{1}<d, \\
\left(\mu_{k 0}\left(x_{1}-d\right), 0\right)^{T}, & x_{1} \geq d,
\end{array}\right.
\end{aligned}
$$

where the dot ' ' denotes the differentiation with respect to the nondimensional time $t$. Let $\mathbf{x}=\left(x_{1}, x_{2}\right)^{T}$; it satisfies that $\dot{x}=\left(v_{1}, v_{2}\right)^{T}$ and $\mathbf{M}$ and $\mathbf{K}$ denote the matrix of mass and the matrix of stiffness in nondimension form, respectively. The state-space discontinuity surfaces $\mathbf{D}_{1}$ and $\mathbf{D}_{2}$ can be expressed as $\quad \mathbf{D}_{1}=\mathbf{x}\left|h^{\mathrm{LC}}(\mathbf{x})=x_{1}+d=0, \quad \mathbf{D}_{2}=\mathbf{x}\right| h^{\mathrm{RC}}$ $(\mathbf{x})=x_{1}-d=0$, where $h^{\mathrm{LC}}(\mathbf{x})$ denote the distance to the left constraint $\mathbf{D}_{1}$ and $h^{\mathrm{RC}}(\mathbf{x})$ denote the distance to the right constraint $\mathbf{D}_{2}$.

2.3. Grazing Periodic Motion. The general solution for nonimpact motions of equation (4) is described in the following form:

$$
\begin{aligned}
x_{i}(t)= & \sum_{j=1}^{2} \varphi_{i j}\left(e^{-\eta_{j} t}\left(a_{j} \cos \omega_{d j} t\right)+A_{j} \sin (\omega t+\tau)\right. \\
& \left.+B_{j} \cos (\omega t+\tau)\right), \\
\dot{x}_{i}(t)= & \sum_{j=1}^{2} \varphi_{i j}\left(e ^ { - \eta _ { j } t } \left(\left(b_{j} \omega_{d j}-a_{j} \eta_{j}\right) \cos \omega_{d j} t\right.\right. \\
& \left.-\left(b_{j} \eta_{j}+a_{j} \omega_{d j}\right) \sin \omega_{d j} t\right)+A_{j} \omega \cos (\omega t+\tau) \\
& \left.-B_{j} \omega \sin (\omega t+\tau)\right),
\end{aligned}
$$

where $\varphi_{i j}$ are the elements of the canonical modal matrix $\Psi$, $\eta_{j}=\xi \omega_{j}^{2}, \quad \omega_{d j}=\sqrt{\omega_{j}^{2}-\eta_{j}^{2}}, \quad\left(\widetilde{f}_{1}, \widetilde{f}_{2}\right)^{T}=\Psi^{T}[1-p p]^{T}, a_{j}$ and $b_{j}$ are the constants of integration that are determined by the initial conditions and system parameter, $A_{j}$ and $B_{j}$ are the constants of amplitude, and their expressions are as follows:

$$
\begin{aligned}
& A_{j}=\widetilde{f}_{j}\left(\frac{\omega_{j}^{2}-\omega^{2}}{\left(\omega_{j}^{2}-\omega^{2}\right)^{2}+\left(2 \eta_{j} \omega\right)^{2}}\right), \\
& B_{j}=\tilde{f}_{j}\left(\frac{-2 \eta_{j} \omega}{\left(\omega_{j}^{2}-\omega^{2}\right)^{2}+\left(2 \eta_{j} \omega\right)^{2}}\right) .
\end{aligned}
$$

The considered system consists of bilateral symmetrical elastic stops; the initial conditions and periodic motion conditions of the orbit with double grazing bifurcation satisfy the following formula:

$$
\begin{aligned}
x_{1}(0) & =x_{10}=d, \\
x_{2}(0) & =x_{20}, \\
v_{1}(0) & =v_{10}=0, \\
v_{2}(0) & =v_{20}, \\
x_{1}\left(\frac{\pi}{\omega}\right) & =-d, \\
x_{1}\left(\frac{2 p \pi}{\omega}\right) & =x_{10}=d, \\
x_{2}\left(\frac{2 p \pi}{\omega}\right) & =x_{20}, \\
v_{1}\left(\frac{2 p \pi}{\omega}\right) & =v_{10}=0, \\
v_{2}\left(\frac{2 p \pi}{\omega}\right) & =v_{20} .
\end{aligned}
$$

By substituting the above conditions into the general solution, we can see that

$$
\begin{gathered}
d^{2}=d_{1}^{2}+d_{2}^{2} \neq 0, \\
\tau=\arctan \left(\frac{d_{1}}{d_{2}}\right),
\end{gathered}
$$

where $d_{1}=A_{1} \varphi_{11}+A_{2} \varphi_{12}, d_{2}=B_{1} \varphi_{11}+B_{2} \varphi_{12}$.

With appropriate system parameters, the motion of the system is periodic. The periodic motion of the system is represented by $(p, q, n)$, the number of periods is represented by $n, p$ denotes the number of collisions between the mass $M_{1}$ and the left side constraint, and $q$ denotes the number of collisions between the mass $M_{1}$ and the right side constraint.

\section{Codimension-One Bifurcation Analysis}

In this section, we will use the COCO-toolbox to study the dynamic behavior, giving the path-following analysis with respect to the frequency of the excitation of the system parameters. The state space of the system is divided into three regions according to the motion state of the oscillator. And then event functions are defined to describe the critical conditions of each mode. We take $\mathbf{u}=\left(x_{1}, \dot{x}_{1}, x_{2}, \dot{x}_{2}\right)^{T} \varepsilon \mathbf{R}^{4}$, as phase variable, and the values of the other nondimensional parameters are fixed as $\lambda=\left(\mu_{m}, d, \mu_{k 1}, \mu_{c}, \xi\right.$, $\left.\mu_{k 2}, f_{2}, \tau\right)=(0.25,0.1,0.1,0.25,0.2,100,0.5,0.9276)$ except for the frequency of the excitation.

The segments needed for numerical simulation in $\mathrm{COCO}$ are shown in Table 1.

In order to better show the dynamic behaviors of the oscillators under the case of elastic constraint, we take the 
TABLE 1: The modes, vector fields, and event functions used for numerical simulation in COCO.

\begin{tabular}{lllc}
\hline Interval & Mode & Vector field & Event function \\
\hline$x_{1} \leq-d$ & Left contact (LC) & $\dot{u}=f_{\mathrm{LC}}(t, \mathbf{u}, \lambda)$ & $h^{\mathrm{LC}}=x_{1}+d$ \\
$-d<x_{1}<d$ & Free flight (FF) & $\dot{u}=f_{\mathrm{FF}}(t, \mathbf{u}, \lambda)$ & $h^{\mathrm{LC}}=x_{1}+d, h^{\mathrm{RC}}=x_{1}-d$ \\
$x_{1} \geq d$ & Right contact (RC) & $\dot{u}=f_{\mathrm{RC}}(t, \mathbf{u}, \lambda)$ & $h^{\mathrm{RC}}=x_{1}-d$ \\
\hline
\end{tabular}

frequency of excitation as a controlling parameter. Because the constraints on both sides of the system are symmetrical, we take the left contact time as an example. And we discuss the bifurcations of the system as the contact time between the mass $M_{1}$ and the left-hand side spring varies with the frequency of external excitation.

In the following bifurcation diagram, the point on the solid line corresponds to a stable orbit, while the solid line on the phase diagram represents a stable solution. The vertical coordinate LTOC in the following bifurcation diagram represents the contact time between the mass $M_{1}$ and the spring on the left.

In Figure 2, we show the result of the numerical continuation of the period-1 solution with respect to the frequency $\omega$. We start continuation from the larger value of $\omega$; at this moment, there is no interaction between the oscillator $M_{1}$ and the stop. Thus, the nonimpacting solution corresponds to a horizontal line with the LTOC being zero in Figure 2 . From here, we can find a stable $(0,0,1)$ solution. In the direction of decreasing $\omega$, we find a grazing bifurcation point where the orbit makes tangential contact with both the left and right constraint surfaces (denoted as DGR1) at $\omega \approx 2.768$ as shown in Figure 3(a), after which the impacting motion occurs. Very close to the grazing point, a saddlenode bifurcation is detected. Here, a Floquet multiplier of the periodic solution crosses the unit circle from the inside, and therefore the stability is lost. The orbit goes to the direction of increasing $\omega$ until the second saddle-node bifurcation SN2 is encountered at $\omega \approx 6.021$. And the solution obtains stability; after this point, the orbit turns to the direction of decreasing $\omega$. If we trace this stable branch, the third saddle-node bifurcation SN3 occurs at $\omega \approx 2.639$, and the orbit loses stability again. If $\omega$ is decreased further, another double grazing bifurcation point DGR2 shown in Figure 3(b) is found. As shown in Figure 2(c), in the direction of increase of $\omega$, the saddle-node bifurcation point SN4 and period-doubling bifurcation point PD1 are detected in turn. And the stability of the periodic solution changes. Along the unstable $(2,1,1)$ orbit, we detect the perioddoubling bifurcation point PD2 and the saddle-node bifurcation point SN5; finally, the stability is lost. This unstable impacting solution is traced further as shown in Figure 2(d). A saddle-node bifurcation point SN6 occurs, and the orbit regains stability. When $\omega$ is increased along the stable $(2,1$, 1) orbit, the period-doubling bifurcation PD3 is found, and the stable orbit becomes unstable $(2,1,1)$ orbit again. By tracing this unstable branch, we can detect the left grazing point where the solution is tangent to the left constraint (denoted as LGR) in $(1,1,1)$ orbit at $\omega \approx 2.132$. If $\omega$ is increased further, a period-doubling bifurcation PD4 in (1, $1,1)$ orbit is encountered at $\omega \approx 2.435$, and the perioddoubling bifurcation makes the $(1,1,1)$ orbit stable. If we trace this period orbit in the direction of increasing the frequency $\omega$, the saddle-node point SN3 is detected. After this point, the branch of $(1,1,1)$ orbit turns to the direction of decreasing $\omega$ until a period-doubling point PD5 appears, and then the solution loses the stability. As shown in Figure 2(e), as $\omega$ is decreased further along the unstable (1, 1, 1) branch, a grazing bifurcation where the orbit makes tangential contact with right constraint (denoted as RGR) is detected, after which the impact between the oscillator $M_{1}$ and the right spring occurs, and then the unstable $(1,2,1)$ branch emerges. In the direction of decreasing the frequency $\omega$, a period-doubling bifurcation PD6 in $(1,2,1)$ orbit makes the $(1,2,1)$ branch stable again. When we decrease $\omega$ further, a saddle-node bifurcation SN7 in $(1,2,1)$ orbit is detected, and the solution loses the stability. The branch has now switched to the direction of increasing the parameter $\omega$. Along this branch, we find another saddle-node bifurcation SN8 in $(1,2,1)$ orbit at $\omega \approx 2.373$. If $\omega$ is decreased further, a period-doubling bifurcation PD7 is detected. Finally, the solution loses stability.

By tracing the period-1 solutions via the continuation platform COCO, we detect eight period-doubling points which give rise to four period-2 branches. The path-following analysis of the period-2 branch with respect to the frequency $\omega$ is shown in Figure 4.

In Figure 4(a), starting from the period-doubling bifurcation point PD1 in $(2,1,1)$ orbit, and an unstable $(4,2,2)$ orbit can be found through period-doubling bifurcation. In the vicinity of the period-doubling bifurcation point PD1, we detect a grazing point LGR1 after which the $(4,2,2)$ orbit becomes $(3,2,2)$ orbit. As $\omega$ is increased, a period-doubling bifurcation PD and a saddle-node bifurcation $\mathrm{SN}$ in $(3,2,2)$ orbit are detected respectively, and the stability of periodic solution changes. If the frequency is reduced to $\omega \approx 2.064 \mathrm{a}$ grazing bifurcation LGR2 in $(3,2,2)$ orbit appears, where the solution makes tangential contact with the left stop, after which the branch of unstable $(3,2,2)$ orbit becomes the branch of unstable $(4,2,2)$ orbit. Later, as $\omega$ is increased, we find another saddle-node bifurcation $\mathrm{SN}$ and period-doubling bifurcation PD in $(4,2,2)$ orbit; finally, the solution becomes stable. Along this stable branch, finally, it returns to the period-doubling bifurcation point PD2 of the period-1 orbit.

We start continuation at the period-doubling bifurcation point PD3 of the period-1 branch, and a period-doubling bifurcation PD is detected when $\omega$ is increased. Below this 


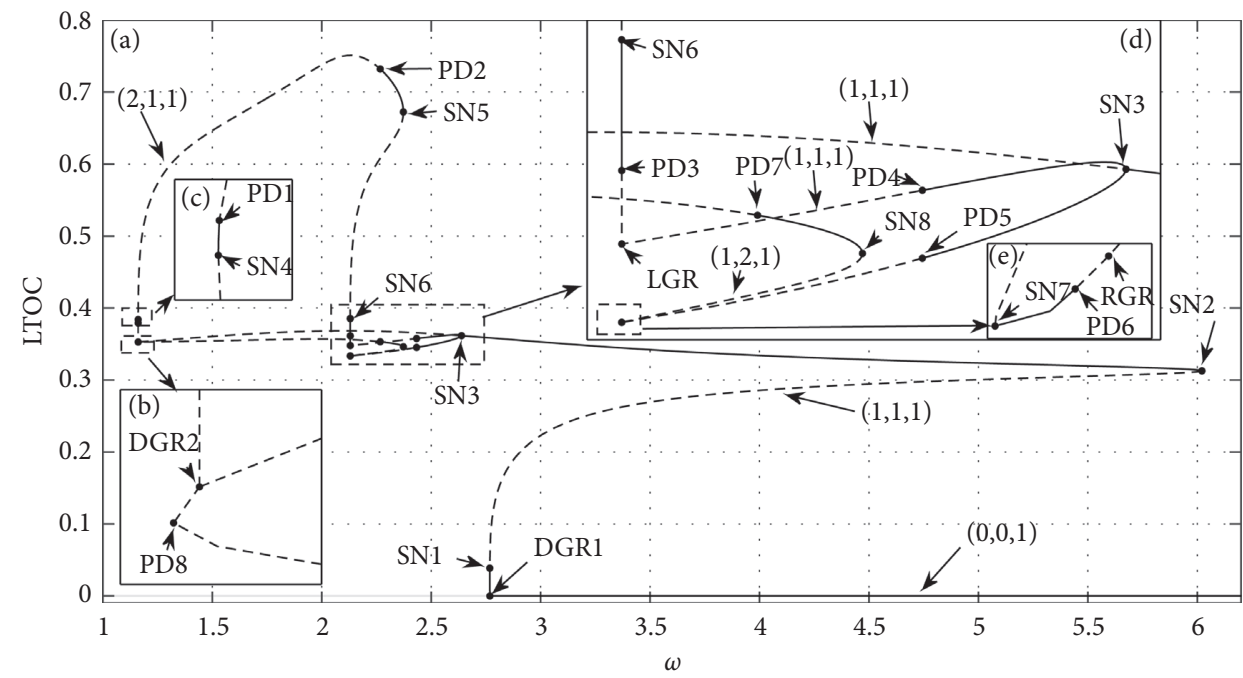

Figure 2: The result of the numerical continuation of the period-1 solution with respect to the frequency $\omega$. The black solid and black dashed orbits denote stable and unstable solutions, respectively. Bifurcation points are marked by black dots.

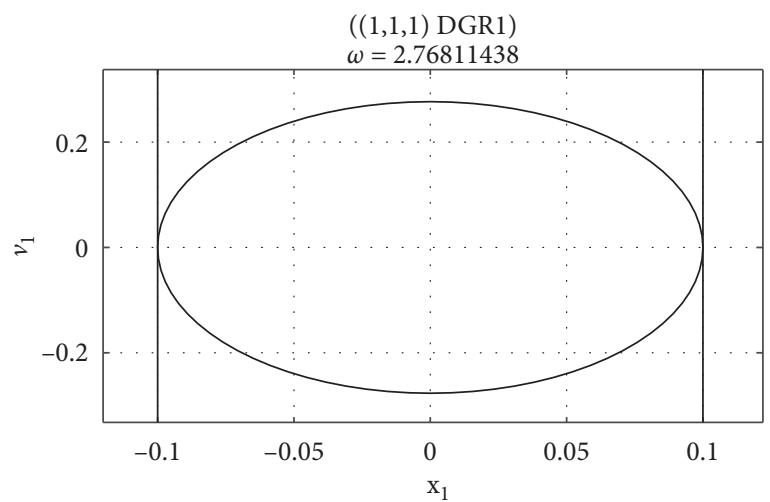

(a)

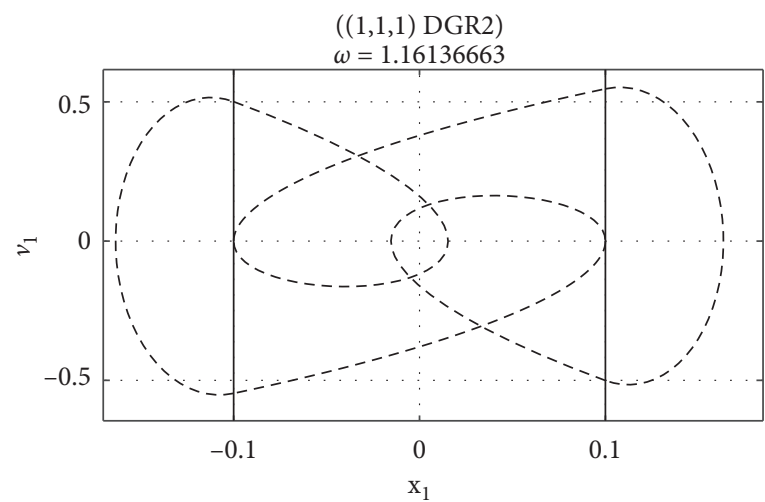

(b)

FIgURE 3: (a) The phase diagram at the double grazing bifurcation point DGR1 of the period-1 orbit detected at $\omega \approx 2.76811438$. (b) The phase diagram at the double grazing bifurcation point DGR2 of the period-1 orbit detected at $\omega \approx 1.16136663$.

point, we find a $(4,2,2)$ branch, which terminates at the grazing bifurcation point LGR3. Along this $(3,2,2)$ orbit, a period-doubling bifurcation point $\mathrm{PD}$ and a saddle-node bifurcation point SN are detected, respectively, and the stability of periodic solution changes. As $\omega$ is increased, we find another grazing bifurcation point LGR4 after which the $(3,2,2)$ orbit becomes $(2,2,2)$ orbit. Finally, the period-2 branch returns to the period-doubling point PD4 of period-1 orbit.

The results of the numerical continuation of other period-2 impacting solutions with respect to the frequency $\omega$ are presented in Figure 4(b). Starting from the perioddoubling bifurcation point PD5 of the period-1 orbit, we can detect a stable branch until the period-doubling bifurcation is reached at $\omega \approx 2.386$, and then the orbit loses stability. When we trace this branch further in the direction of decreasing the frequency $\omega$, a grazing bifurcation RGR1 is found, where the $(2,2,2)$ orbit becomes $(2,3,2)$ orbit. As $\omega$ is decreased, a saddle-node bifurcation occurs. Below this value, we find a small window of stability, which ends at the period-doubling bifurcation point $\mathrm{PD}$, and then the branch loses stability. Along this unstable branch, another grazing bifurcation point RGR2 is detected where the $(2,3,2)$ orbit becomes the $(2,4,2)$ orbit. Finally, the orbit returns to the period-doubling bifurcation point PD6 of the period-1 orbit. A similar behavior can be observed around another period-2 orbit, which is shown in the upper part of Figure 4(b).

\section{Codimension-Two Grazing Bifurcation}

4.1. The Zero-Time-Discontinuity Mapping. Now suppose that $\mathbf{x}^{* 1}$ and $\mathbf{x}^{* 2}$ are symmetrical grazing points of a statespace trajectory with the constraints $\mathbf{D}_{1}$ and $\mathbf{D}_{2}$, such that 


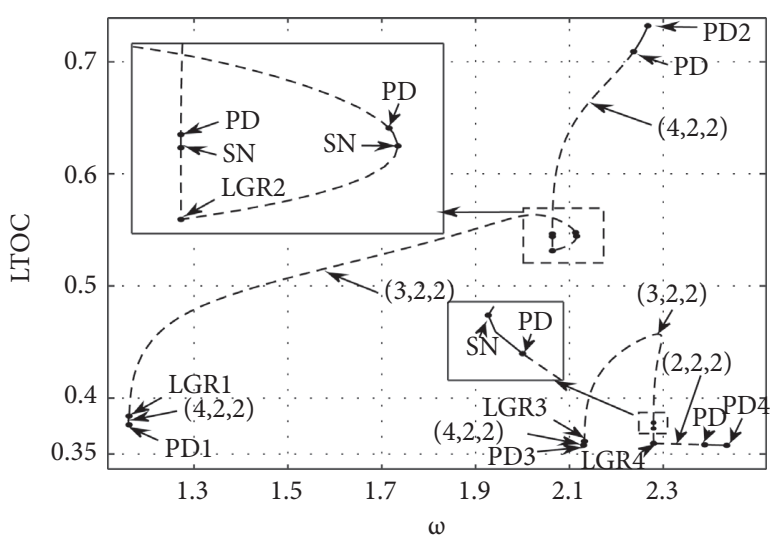

(a)

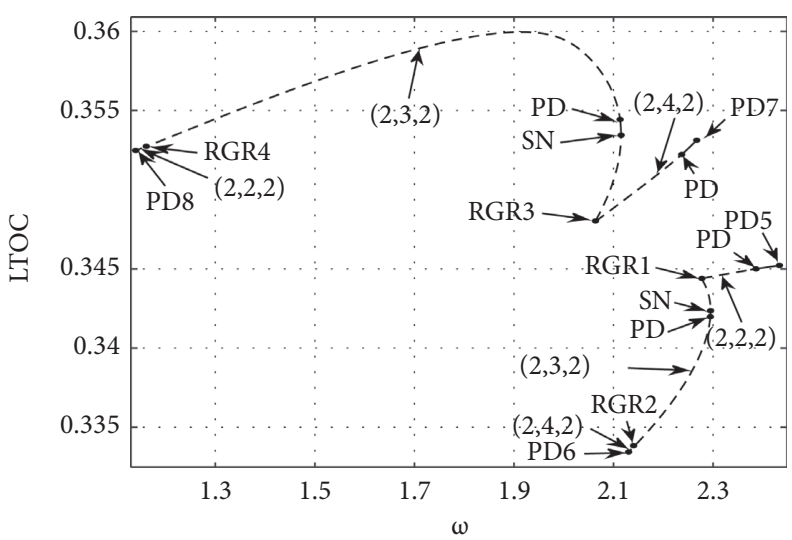

(b)

FIgURE 4: The result of the numerical continuation of the period-2 solution with respect to the frequency $\omega$. The solid and dashed orbits denote stable and unstable solutions, respectively. Bifurcation points are marked by black dots. (a) Two orbits of period-2 originated from the period-doubling bifurcation points PD1 and PD3 of the period-1 orbit, respectively. (b) Two orbits of period-2 originated from the period-doubling bifurcation points PD5 and PD7 of the period-1 orbit, respectively.

$$
\begin{aligned}
h^{\mathrm{LC}}\left(\mathbf{x}^{* 1}\right) & =0, h^{\mathrm{RC}}\left(\mathbf{x}^{* 2}\right)=0, v_{1}^{* 1}=h^{P 1}\left(\mathbf{x}^{* 1}\right) \\
& =\frac{\partial h^{\mathrm{LC}}\left(\mathbf{x}^{* 1}\right)}{\partial t}=0, \\
v_{1}^{* 2} & =h^{P 2}\left(\mathbf{x}^{* 2}\right)=\frac{\partial h^{\mathrm{RC}}\left(\mathbf{x}^{* 2}\right)}{\partial t}=0, a_{1}^{* 1} \\
& =\frac{\partial^{2} h^{\mathrm{LC}}\left(\mathbf{x}^{* 1}\right)}{\partial t^{2}}<0, a_{2}^{* 2}=\frac{\partial^{2} h^{\mathrm{RC}}\left(\mathbf{x}^{* 2}\right)}{\partial t^{2}}<0 .
\end{aligned}
$$

We define two Poincare surfaces $\Pi_{1}$ and $\Pi_{2}$ : $\Pi_{1}=\mathbf{x} \varepsilon \mathbf{R}^{5}\left|\theta=\theta^{*}, \Pi_{2}=\mathbf{x} \varepsilon \mathbf{R}^{5}\right| \theta=\theta^{*}+\pi$, where the state vector $\mathbf{x}=\left(x_{1}, v_{1}, x_{2}, v_{2}, \theta\right)^{T}, \theta=\omega t+\tau$, the modulus of $\theta$ being $2 \pi, \theta^{*}=\tau^{*}$, such that $\mathbf{x}^{* 1}$ and $\mathbf{x}^{* 2}$ correspond to a transversal intersection of a state-space trajectory with Poincare surfaces $\Pi_{1}$ and $\Pi_{2}$, respectively, and $\Phi\left(\mathbf{x}^{* 1},(t+T) / 2\right)=\Phi\left(\mathbf{x}^{* 2}, t\right), \Phi\left(\mathbf{x}^{* 2},(t+T) / 2\right)=\Phi\left(\mathbf{x}^{* 1}, t\right)$. Thus, we can introduce two local Poincare maps $\mathbf{P}_{1 \text { smooth }}: \Pi_{1} \longrightarrow \Pi_{2}$ and $\mathbf{P}_{2 \text {,smooth }}: \Pi_{2} \longrightarrow \Pi_{1}$, such that $\mathbf{P}_{1 \text {,smooth }}\left(\mathbf{x}^{* 1}\right)=\mathbf{x}^{* 2}$ and $\mathbf{P}_{2 \text {,smooth }}\left(\mathbf{x}^{* 2}\right)=\mathbf{x}^{* 1}$.

According to the discontinuity mapping of the rigid constraint system introduced by Nordmark [4], the discontinuity mapping is extended to the elastic unilateral constraint system. As shown in Figure 5, there is a grazing trajectory with the discontinuous boundary. Consider the point $\mathbf{x}_{1}$ near the grazing point $\mathbf{x}^{*}$; therefore, it can be seen that the trajectory $\left(\mathbf{x}_{1}, \mathbf{x}_{2}, \mathbf{x}_{3}, \mathbf{x}_{4}\right)$ passes through the discontinuous boundary. From the reverse direction of its trajectory, the time required to move from the point $\mathbf{x}_{3}$ to the point $\mathbf{x}_{0}$ is the same as that from the point $\mathbf{x}_{1}$ to the point $\mathbf{x}_{3}$. So, we assume that there is an instantaneous jump from $\mathbf{x}_{1}$ to $\mathbf{x}_{0}$; then, the zero-time-discontinuity mapping can be defined as the mapping from $\mathbf{x}_{1}$ to $\mathbf{x}_{0}$. For the specific process of discontinuity-mapping expression of the elastic constraint system, see [4].

Based on the previous analysis, we extend the discontinuity mapping of elastic constraint systems from unilateral constraint to bilateral constraints. The two corresponding

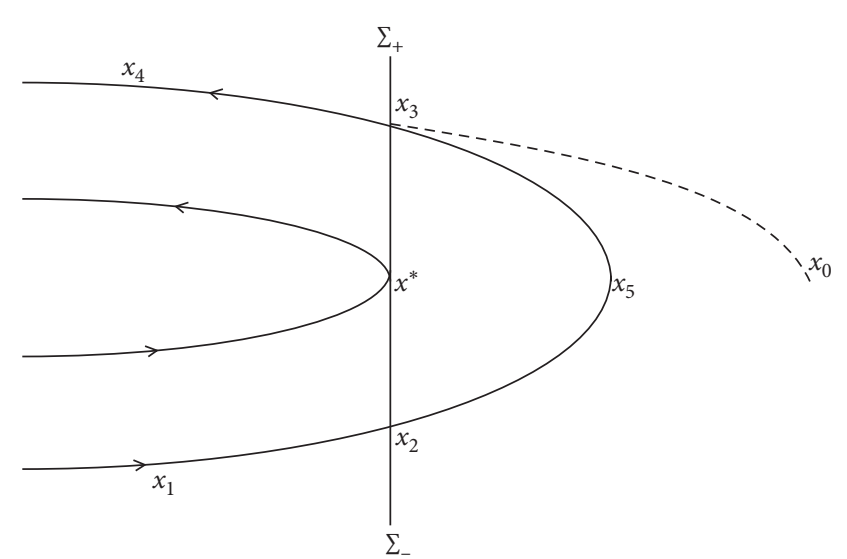

FIgURE 5: The sketch map of the zero-time-discontinuity mapping near the grazing trajectory.

zero-time-discontinuity mappings $\mathbf{Z D M}_{1}$ and $\mathbf{Z D M}_{2}$ near two grazing points $\mathbf{x}^{* 1}$ and $\mathbf{x}^{* 2}$ can be written, respectively, as follows:

$$
\begin{aligned}
& \mathbf{Z D M}_{1}= \begin{cases}\mathbf{x}, & h_{\min }^{\mathrm{LC}}(\mathbf{x}) \geq 0, \\
\mathbf{x}^{* 1}+\frac{8}{3} \sqrt{\frac{-2}{a_{1}^{* 1}}}\left(h_{\mathrm{min}}^{\mathrm{LC}}\right)^{(3 / 2)} \mathbf{Q}, & h_{\min }^{\mathrm{LC}}(\mathbf{x})<0,\end{cases} \\
& \mathbf{Z D M}_{2}= \begin{cases}\mathbf{x}, & h_{\max }^{\mathrm{RC}}(\mathbf{x})<0, \\
\mathbf{x}^{* 2}+\frac{8}{3} \sqrt{\frac{-2}{a_{1}^{* 2}}}\left(h_{\mathrm{max}}^{\mathrm{RC}}\right)^{(3 / 2)} \mathbf{Q}, & h_{\max }^{\mathrm{RC}}(\mathbf{x}) \geq 0,\end{cases}
\end{aligned}
$$

where $\mathbf{Q}=\left(0,-\mu_{k 0}, 0,0,0\right)^{T}, h_{\max }^{\mathrm{RC}}(\mathbf{x})$ represents the maximum displacement across the right collision surface, $h_{\min }^{\mathrm{LC}}(\mathbf{x})$ represents the minimum displacement across the left collision surface. 
The two zero-time-discontinuity mappings $\mathbf{Z} \mathbf{D M}_{1}$ and $\mathbf{Z} \mathbf{D M}_{2}$ are defined in the vicinity of the grazing points $\mathbf{x}^{* 1}$ and $\mathbf{x}^{* 2}$, respectively, so the Poincare mapping $\mathbf{P}$ near the grazing trajectory has the following form:

$$
\mathbf{P}=\mathbf{P}_{2 \text {,smooth }} \circ \mathbf{Z} \mathbf{D M}_{2} \circ \mathbf{P}_{1, \text { smooth }} \circ \mathbf{Z} \mathbf{D M}_{1} .
$$

4.2. The Poincare Mapping. The mapping $\mathbf{P}_{1, \text { smooth }}$ can be expanded near the grazing point $\mathbf{x}^{* 1}$ as follows:

$$
\begin{aligned}
\mathbf{P}_{1, \text { smooth }}(\mathbf{x})= & \mathbf{P}_{1, \text { smooth }}\left(\mathbf{x}^{* 1}\right)+\mathbf{P}_{1, \text { smooth }, x}\left(\mathbf{x}^{* 1}\right)\left(\mathbf{x}-\mathbf{x}^{* 1}\right) \\
& + \text { h.o.t } \\
= & \mathbf{x}^{* 2}+\mathbf{P}_{1, \text { smooth }, x}\left(\mathbf{x}^{* 1}\right)\left(\mathbf{x}-\mathbf{x}^{* 1}\right)+\text { h.o.t. }
\end{aligned}
$$

Similarly, we expand the event function $h^{\mathrm{LC}}$ near $\mathbf{x}^{* 1}$ and then $h^{\mathrm{LC}}$ have the following form:

$$
h^{\mathrm{LC}}(\mathbf{x})=h^{\mathrm{LC}}\left(\mathbf{x}^{* 1}\right)+h_{x}^{\mathrm{LC}}\left(\mathbf{x}^{* 1}\right)\left(\mathbf{x}-\mathbf{x}^{* 1}\right)+\text { h.o.t. }
$$

As the point $\mathbf{x}^{* 1}$ is on the impact surface $\mathbf{D}_{1}$, it needs to satisfy $h^{\mathrm{LC}}\left(\mathbf{x}^{* 1}\right)=0$, such that (14) is expressed as

$$
h^{\mathrm{LC}}(\mathbf{x})=h_{x}^{\mathrm{LC}}\left(\mathbf{x}^{* 1}\right)\left(\mathbf{x}-\mathbf{x}^{* 1}\right)+\text { h.o.t, }
$$

And, consequently,

$$
\mathbf{P}_{1}=\mathbf{P}_{1, \text { smooth }}(\mathbf{x})^{\circ} \mathbf{Z D M}_{1}(\mathbf{x})= \begin{cases}\mathbf{x}^{* 2}+\mathbf{P}_{1, \text { smooth }, \mathrm{x}}\left(\mathbf{x}^{* 1}\right)\left(\mathbf{x}-\mathbf{x}^{* 1}\right)+\text { h.o.t, } & \mathrm{h}_{\min }^{\mathrm{LC}}(\mathbf{x}) \geq 0, \\ \mathbf{x}^{* 2}+\mathbf{P}_{1, \text { smooth }, \mathrm{x}}\left(\mathrm{x}^{* 1}\right) \frac{8}{3} \sqrt{\frac{-2}{\mathrm{a}_{1}^{* 1}}}\left(\mathrm{~h}_{\text {min }}^{\mathrm{LC}}\right)^{(3 / 2)} \mathbf{Q}+\text { h.o.t, }, & \mathrm{h}_{\text {min }}^{\mathrm{LC}}(\mathbf{x})<0 .\end{cases}
$$

Similar to the above analysis, we have

$$
\mathbf{P}_{2}=\mathbf{P}_{2, \text { smooth }}(\mathbf{x})^{\circ} \mathbf{Z D M}_{2}(\mathbf{x})= \begin{cases}\mathbf{x}^{* 1}+\mathbf{P}_{2, \text { smooth }, \mathrm{x}}\left(\mathbf{x}^{* 2}\right)\left(\mathbf{x}-\mathbf{x}^{* 2}\right)+\text { h.o.t, } & \mathrm{h}_{\max }^{\mathrm{RC}}(\mathbf{x}) \leq 0, \\ \mathbf{x}^{* 1}+\mathbf{P}_{2, \text { smooth }, \mathrm{x}}\left(\mathbf{x}^{* 2}\right) \frac{8}{3} \sqrt{\frac{-2}{\mathrm{a}_{1}^{* 2}}\left(\mathrm{~h}_{\max }^{\mathrm{RC}}\right)^{(3 / 2)} \mathrm{Q}+\text { h.o.t, },} & \mathrm{h}_{\max }^{\mathrm{RC}}(\mathbf{x})>0 .\end{cases}
$$

\subsection{Stability and Codimension-Two Grazing Bifurcation}

4.3.1. Conditions of Codimension-Two Grazing Bifurcation. If the starting point near the grazing point, which starts from either the impact side or nonimpacting side, converges to the grazing point after the iteration of mappings (16) and (17), the grazing periodic orbit is stable. For an impact point $\mathbf{x}$ in the neighborhood of the grazing point $\mathbf{x}^{* 1}$, it satisfies $h_{x}^{\mathrm{LC}}\left(\mathbf{x}^{* 1}\right)\left(\mathbf{x}-\mathbf{x}^{* 1}\right)<0$. After the iteration of the mapping $\mathbf{P}_{1}$, it reaches the nonimpacting side, which satisfies

$$
\begin{aligned}
h_{x}^{\mathrm{RC}}\left(\mathbf{x}^{* 2}\right)\left(P_{1}(\mathbf{x})-\mathbf{x}^{* 2}\right)=h_{x}^{\mathrm{RC}}\left(\mathbf{x}^{* 2}\right) \mathbf{P}_{1, \text { smooth }, x}\left(\mathbf{x}^{* 1}\right) \\
\cdot \frac{8}{3} \sqrt{\frac{-2}{a_{1}^{* 1}}}\left(h_{\min }^{\mathrm{LC}}\right)^{(3 / 2)} \mathbf{Q}<0 ;
\end{aligned}
$$

that is,

$$
h_{x}^{\mathrm{RC}}\left(\mathbf{x}^{* 2}\right) \mathbf{P}_{1, \text { smooth }, x}\left(\mathbf{x}^{* 1}\right) \mathbf{Q}<0 .
$$

And after the iteration of the mapping $\mathbf{P}_{2}$, the oscillator collides with the discontinuity surface $\mathbf{D}_{1}$ again, which satisfies

$$
\begin{aligned}
h_{x}^{\mathrm{LC}}\left(\mathbf{x}^{* 1}\right) & \left(\mathbf{P}_{2}\left(\mathbf{P}_{1}(\mathbf{x})\right)-\mathbf{x}^{* 1}\right) \\
= & h_{x}^{\mathrm{LC}}\left(\mathbf{x}^{* 1}\right) \mathbf{P}_{2, \text { smooth }, x}\left(\mathbf{x}^{* 2}\right) \mathbf{P}_{1, \text { smooth }, x}\left(x^{* 1}\right) \\
& \cdot \frac{8}{3} \sqrt{\frac{-2}{a_{1}^{*}}}\left(h_{\text {min }}^{\mathrm{LC}}\right)^{(3 / 2)} \mathbf{Q}<0 ;
\end{aligned}
$$

that is,

$$
h_{x}^{\mathrm{LC}}\left(\mathbf{x}^{* 1}\right) \mathbf{P}_{2, \text { smooth }, x}\left(\mathbf{x}^{* 2}\right) \mathbf{P}_{1, \text { smooth }, x}\left(\mathbf{x}^{* 1}\right) \mathbf{Q}<0 .
$$

This shows that if the oscillator collides with the impact surface $\mathbf{D}_{1}$ again after iteration, the impact will be persisted, which results in a large stretch of the orbit in a certain direction. Therefore, the grazing periodic orbit is unstable.

Based on the above analysis, if 


$$
\begin{array}{r}
h_{x}^{\mathrm{RC}}\left(\mathbf{x}^{* 2}\right) \mathbf{P}_{1, \text { smooth }, x}\left(\mathbf{x}^{* 1}\right)\left(\mathbf{P}_{2, \text { smooth }, x}\left(\mathbf{x}^{* 2}\right) \mathbf{P}_{1, \text { smooth }, x}\left(\mathbf{x}^{* 1}\right)\right)^{(j-1)} \mathbf{Q}<0, \\
h_{x}^{\mathrm{LC}}\left(\mathbf{x}^{* 1}\right)\left(\mathbf{P}_{2, \text { smooth }, x}\left(\mathbf{x}^{* 2}\right) \mathbf{P}_{1, \text { smooth }, x}\left(\mathbf{x}^{* 1}\right)\right)^{i} \mathbf{Q}<0, \\
2 \leq j \leq i,
\end{array}
$$

it means that the oscillator changes from the impact side to the nonimpacting side after several iterations but finally collides with the impact surface $\mathbf{D}_{1}$ again and then the impact will be perpetuated. Therefore, the grazing periodic orbit loses stability.

Similarly, if

$$
\begin{array}{r}
h_{x}^{\mathrm{RC}}\left(\mathbf{x}^{* 2}\right) \mathbf{P}_{1, \text { smooth }, x}\left(\mathbf{x}^{* 1}\right) \mathbf{Q}>0, \\
h_{x}^{\mathrm{RC}}\left(\mathbf{x}^{* 2}\right) \mathbf{P}_{1, \text { smooth }, x}\left(\mathbf{x}^{* 1}\right) \mathbf{P}_{2, \text { smooth }, x}\left(\mathbf{x}^{* 2}\right) \mathbf{Q}>0,
\end{array}
$$

the oscillator collides with the impact surface $\mathbf{D}_{2}$ again after iteration, and the impact will be perpetuated; then, the grazing periodic orbit is unstable.

According to the above analysis, if

$$
\begin{array}{r}
h_{x}^{\mathrm{RC}}\left(\mathbf{x}^{* 2}\right) \mathbf{P}_{1, \text { smooth }, x}\left(\mathbf{x}^{* 1}\right)\left(\mathbf{P}_{2, \text { smooth }, x}\left(\mathbf{x}^{* 2}\right) \mathbf{P}_{1, \text { smooth }, x}\left(\mathbf{x}^{* 1}\right)\right)^{i} \mathbf{Q}>0, \\
h_{x}^{\mathrm{RC}}\left(\mathbf{x}^{* 2}\right)\left(\mathbf{P}_{1, \text { smooth }, x}\left(\mathbf{x}^{* 1}\right) \mathbf{P}_{2, \text { smooth }, x}\left(\mathbf{x}^{* 2}\right)\right)^{(j+1)} \mathbf{Q}>0, \\
1 \leq i \leq j,
\end{array}
$$

that indicates the oscillator changes from the impact side to the nonimpacting side after several iterations but finally impacts the impact surface $\mathbf{D}_{2}$ again and the impact will be perpetuated. Thus, the grazing periodic orbit loses stability.

In the same way, if

$$
h_{x}^{\mathrm{RC}}\left(\mathbf{x}^{* 2}\right) \mathbf{P}_{1, \text { smooth }, x}\left(\mathbf{x}^{* 1}\right) \mathbf{Q}>0, \quad h_{x}^{\mathrm{LC}}\left(\mathbf{x}^{* 1}\right) \mathbf{P}_{2, \text { smooth }, x}\left(\mathbf{x}^{* 2}\right) \mathbf{Q}<0,
$$

then the oscillator impacts the impact surface $\mathbf{D}_{1}$ and $\mathbf{D}_{2}$ after iteration. If

$$
\begin{array}{r}
h_{x}^{\mathrm{RC}}\left(\mathbf{x}^{* 2}\right) \mathbf{P}_{1, \text { smooth }, x}\left(\mathbf{x}^{* 1}\right)\left(\mathbf{P}_{2, \text { smooth }, x}\left(\mathbf{x}^{* 2}\right) \mathbf{P}_{1, \text { smooth }, x}\left(\mathbf{x}^{* 1}\right)\right)^{i} \mathbf{Q}>0, \\
h_{x}^{\mathrm{LC}}\left(\mathbf{x}^{* 1}\right) \mathbf{P}_{2, \text { smooth }, x}\left(\mathbf{x}^{* 2}\right)\left(\mathbf{P}_{1, \text { smooth }, x}\left(\mathbf{x}^{* 1}\right) \mathbf{P}_{2, \text { smooth }, x}\left(\mathbf{x}^{* 2}\right)\right)^{j} \mathbf{Q}<0, \\
1 \leq i \leq j,
\end{array}
$$

this means that the oscillator collides again with the discontinuity surfaces $\mathbf{D}_{1}$ and $\mathbf{D}_{2}$ after several iterations, and the impact will be perpetuated. Thus, the grazing periodic orbit loses stability.
According to the above analysis, four special cases can be obtained as follows:

$$
\begin{array}{r}
h_{x}^{\mathrm{LC}}\left(\mathbf{x}^{* 1}\right)\left(\mathbf{P}_{2, \text { smooth }, x}\left(\mathbf{x}^{* 2}\right) \mathbf{P}_{1, \text { smooth }, x}\left(\mathbf{x}^{* 1}\right)\right)^{i} \mathbf{Q}=0, \\
h_{x}^{\mathrm{LC}}\left(\mathbf{x}^{* 1}\right) \mathbf{P}_{2, \text { smooth }, x}\left(\mathbf{x}^{* 2}\right)\left(\mathbf{P}_{1, \text { smooth }, x}\left(\mathbf{x}^{* 1}\right) \mathbf{P}_{2, \text { smooth }, x}\left(\mathbf{x}^{* 2}\right)\right)^{j} \mathbf{Q}=0, \\
h_{x}^{\mathrm{RC}}\left(\mathbf{x}^{* 2}\right) \mathbf{P}_{1, \text { smooth }, x}\left(\mathbf{x}^{* 1}\right)\left(\mathbf{P}_{2, \text { smooth }, x}\left(\mathbf{x}^{* 2}\right) \mathbf{P}_{1, \text { smooth }, x}\left(\mathbf{x}^{* 1}\right)\right)^{i} \mathbf{Q}=0, \\
h_{x}^{\mathrm{RC}}\left(\mathbf{x}^{* 2}\right)\left(\mathbf{P}_{1, \text { smooth }, x}\left(\mathbf{x}^{* 1}\right) \mathbf{P}_{2, \text { smooth }, x}\left(\mathbf{x}^{* 2}\right)\right)^{(j+1)} \mathbf{Q}=0, \\
0 \leq i, 0 \leq j .
\end{array}
$$

Based on the definition in the literature [24], the points corresponding to the above four cases are the codimension- two grazing bifurcation points. Let $\xi_{n}=0(n=1,2,3,4)$ express the condition that the codimension-two grazing 
bifurcation points satisfy; then, it can be written in four cases as follows:

(H1) $\quad \xi_{1}=h_{x}^{\mathrm{LC}}\left(\mathbf{x}^{* 1}\right)\left(\mathbf{P}_{2, \text { smooth }, \mathrm{x}}\left(\mathbf{x}^{* 2}\right) \mathbf{P}_{1, \text { smooth }, x}\left(\mathbf{x}^{* 1}\right)\right)^{i}$ $\mathbf{Q}=0$

(H2) $\quad \xi_{2}=h_{x}^{\mathrm{LC}}\left(\mathbf{x}^{* 1}\right) \mathbf{P}_{2, \text { smooth }, x}\left(\mathbf{x}^{* 2}\right)\left(\mathbf{P}_{1, \text { smooth }, x}\left(\mathbf{x}^{* 1}\right)\right.$ $\left.\mathbf{P}_{2, \text { smooth }, x}\left(\mathbf{x}^{* 2}\right)\right)^{j} \mathbf{Q}=0$

(H3) $\quad \xi_{3}=h_{x}^{\mathrm{RC}}\left(\mathbf{x}^{* 2}\right) \mathbf{P}_{1, \text { smooth }, x}\left(\mathbf{x}^{* 1}\right)\left(\mathbf{P}_{2, \text { smooth }, x}\left(\mathbf{x}^{* 2}\right)\right.$ $\left.\mathbf{P}_{1, \text { smooth }, x}\left(\mathbf{x}^{* 1}\right)\right)^{i} \mathbf{Q}=0$

$$
\begin{aligned}
& (\mathrm{H} 4) \quad \xi_{4}=h_{x}^{\mathrm{RC}}\left(\mathbf{x}^{* 2}\right)\left(\mathbf{P}_{1, \text { smooth }, x}\left(\mathbf{x}^{* 1}\right) \mathbf{P}_{2, \text { smooth }, x}\right. \\
& \left.\left(\mathbf{x}^{* 2}\right)\right)^{(j+1)} \mathbf{Q}=0(0 \leq i, 0 \leq j)
\end{aligned}
$$

Next, we take the third case (H3) as an example to simplify the calculation formula:

$$
\begin{aligned}
& \mathbf{P}_{1, \text { smooth }, x}\left(\mathbf{x}^{* 1}\right)\left(\mathbf{P}_{2, \text { smooth }, x}\left(\mathbf{x}^{* 2}\right) \mathbf{P}_{1, \text { smooth }, x}\left(\mathbf{x}^{* 1}\right)\right)^{n} \\
& =\left(\begin{array}{ccccc}
P_{11}^{n+1} & P_{12}^{n+1} & P_{13}^{n+1} & P_{14}^{n+1} & P_{15}^{n+1} \\
P_{21}^{n+1} & P_{22}^{n+1} & P_{23}^{n+1} & P_{24}^{n+1} & P_{25}^{n+1} \\
P_{31}^{n+1} & P_{32}^{n+1} & P_{33}^{n+1} & P_{34}^{n+1} & P_{35}^{n+1} \\
P_{41}^{n+1} & P_{42}^{n+1} & P_{43}^{n+1} & P_{44}^{n+1} & P_{45}^{n+1} \\
P_{51}^{n+1} & P_{52}^{n+1} & P_{53}^{n+1} & P_{54}^{n+1} & P_{55}^{n+1}
\end{array}\right)
\end{aligned}
$$

Thus,

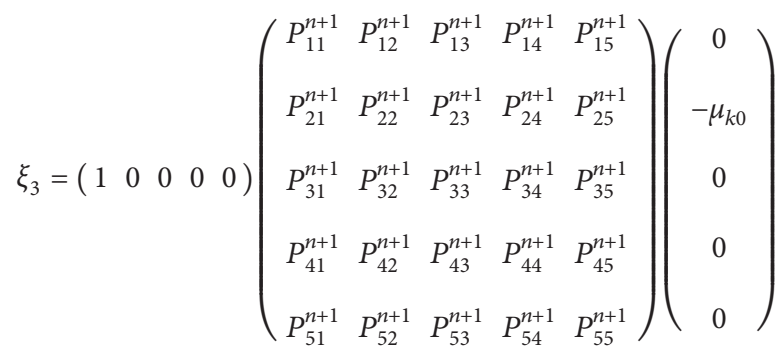

$$
\begin{aligned}
& =-\mu_{k 0} P_{12}^{(n+1)} .
\end{aligned}
$$

Similarly, we can simplify the calculation formulae of codimension-two bifurcation points for the other three cases (H1), (H2), and (H4). Since the system is symmetrically constrained, we can get that $\xi_{1}$ and $\xi_{4}, \xi_{2}$ and $\xi_{3}$ are the same results, respectively, after simplification. The corresponding results are as follows:

$$
\begin{aligned}
& \xi_{1}=\xi_{4}=-\mu_{k 0} P_{12}^{(n)}, \\
& \xi_{2}=\xi_{3}=-\mu_{k 0} P_{12}^{(n+1)},
\end{aligned}
$$

where $P_{12}^{(n)}$ and $P_{12}^{(n+1)}$ denote the $(1,2)$ elements of matrices $\left(\mathbf{P}_{2, \text { smooth }, x}\left(\mathbf{x}^{* 2}\right) \mathbf{P}_{1, \text { smooth }, x}\left(\mathbf{x}^{* 1}\right)\right)^{n}$ and $\mathbf{P}_{1, \text { smooth }, x}\left(\mathbf{x}^{* 1}\right)$ $\left(\mathbf{P}_{2, \text { smooth }, x}\left(\mathbf{x}^{* 2}\right) \mathbf{P}_{1, \text { smooth }, x}\left(\mathbf{x}^{* 1}\right)\right)^{n}$, respectively.

4.3.2. Numerical Simulation. According to the theoretical results deduced above, the existence condition of codimension-two grazing bifurcation points is $\xi_{n}=0(n=1,2,3$,

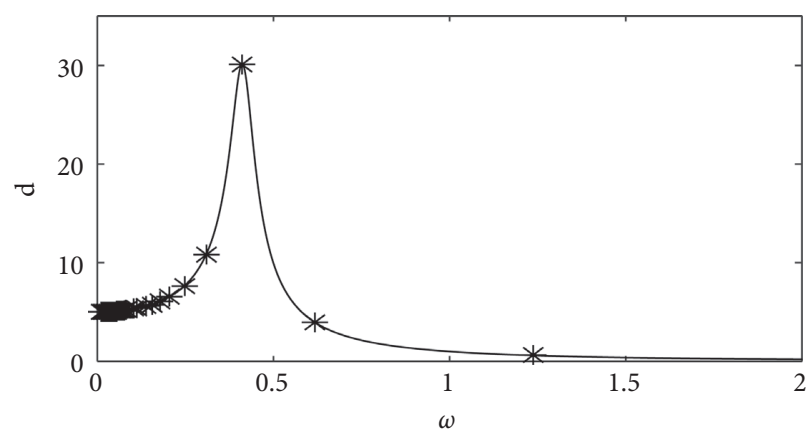

FIgURE 6: The diagram of codimension-two grazing bifurcation points. The black solid line represents the grazing curve, and the points on the line represent the codimension-two grazing bifurcation points.

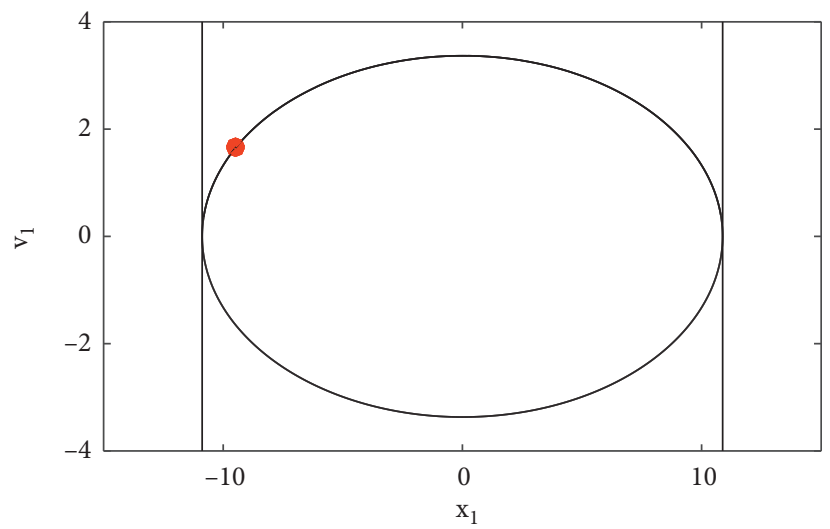

FIgURe 7: A grazing periodic trajectory of oscillator $M_{1}$.

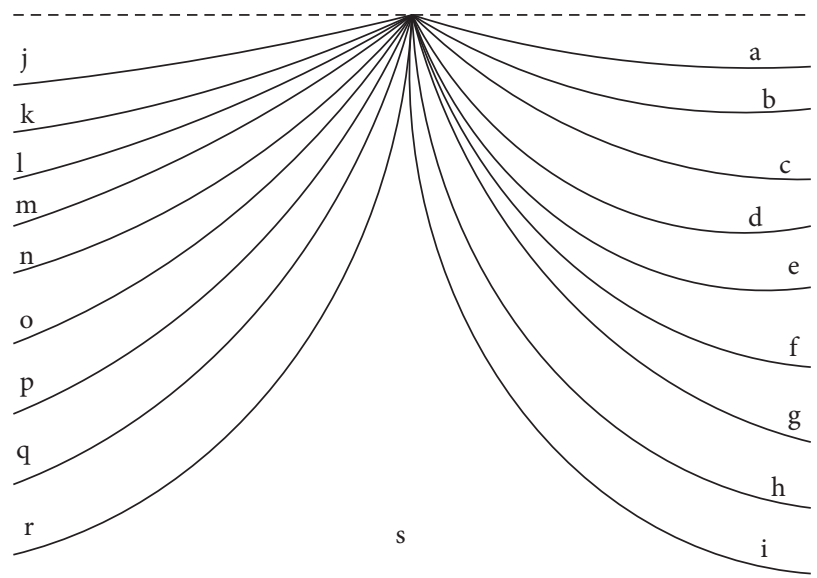

FIGURE 8: Schematic of unfolding diagram near a codimension-two grazing bifurcation point.

4). If the first case (H1) is taken as an example, then the codimension-two grazing bifurcation points correspond to $\xi_{1}=-\mu_{k 0} P_{12}^{n}=0$. Taking $\mu_{k}=0.25, \mu_{m}=0.25, \zeta=0.2, n=1$, when $\omega=0.1376,0.159,0.2091,0.3096,0.6189, \ldots$, we have $\xi_{1}=0$. The grazing curve is shown in Figure 6. The points for which $\xi_{1}=0$ are indicated by ' $*$ ', which are the 


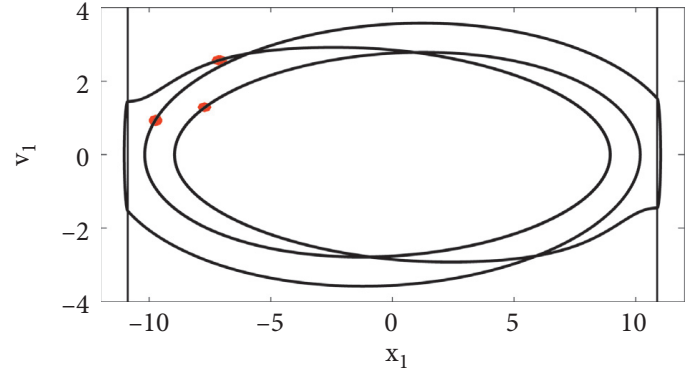

(a)

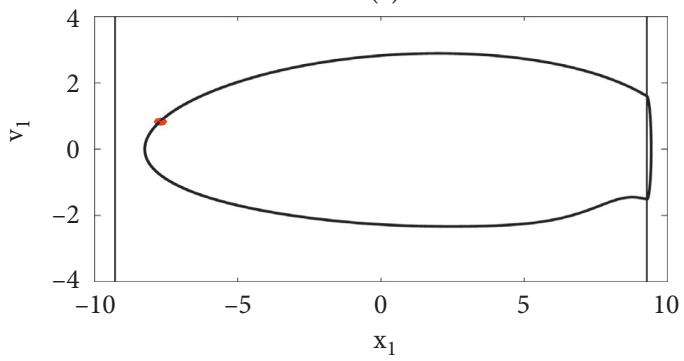

(c)

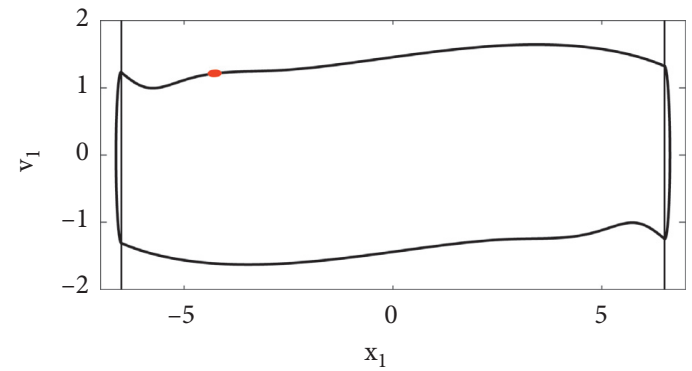

(e)

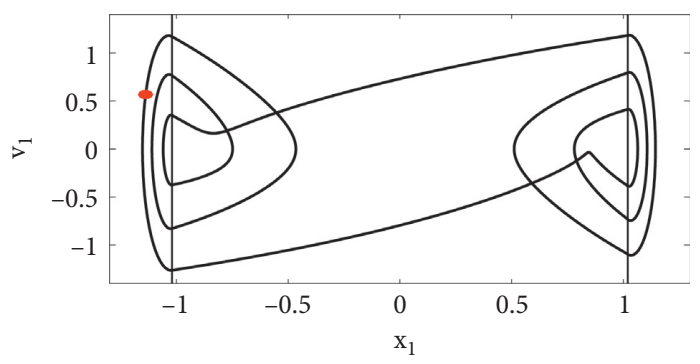

(g)

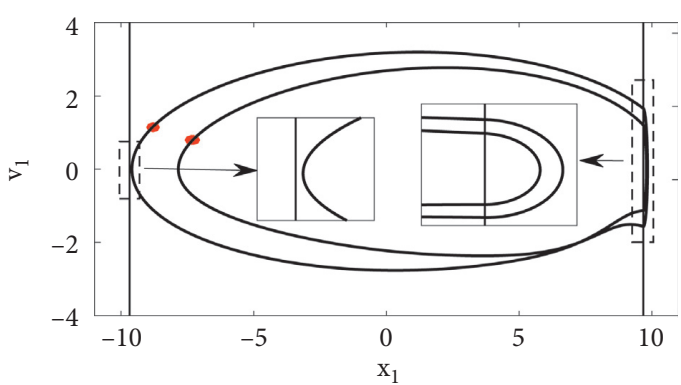

(b)

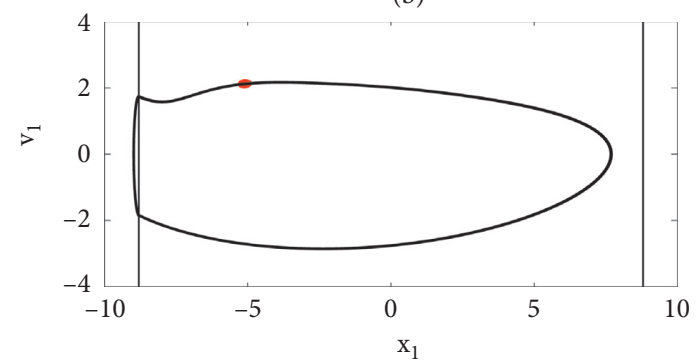

(d)

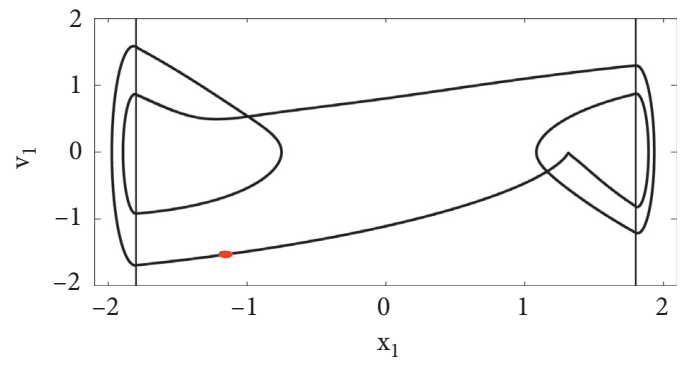

(f)

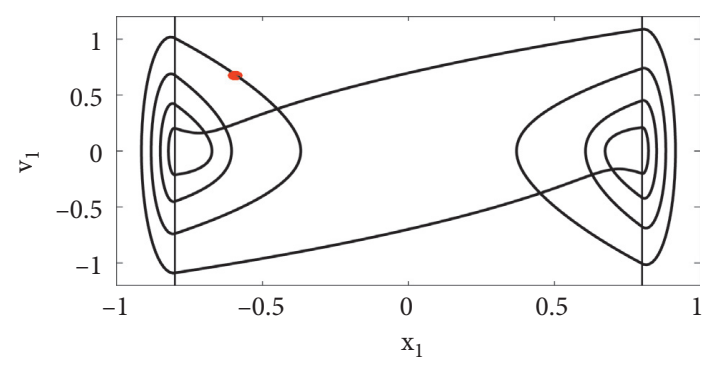

(h)

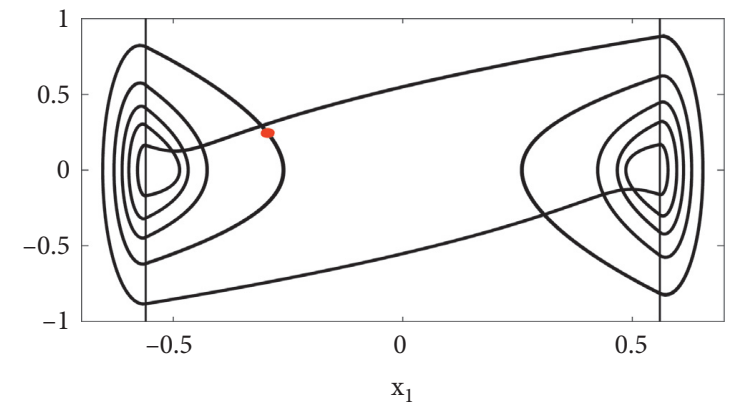

(i)

FIgURE 9: The phase diagrams for periodic motions in regions (a)-(i) of Figure 8. Poincare sections are marked by red dots on the phase planes. 


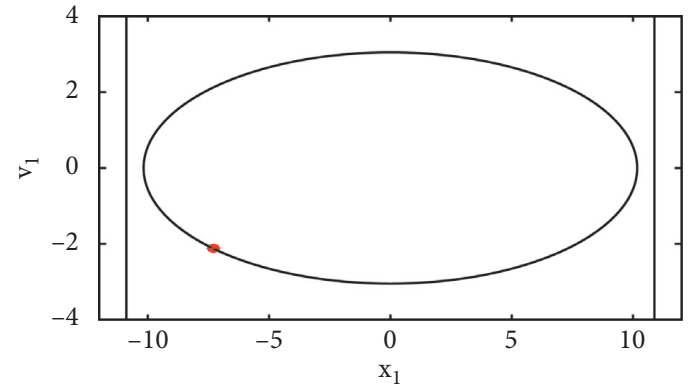

(a)

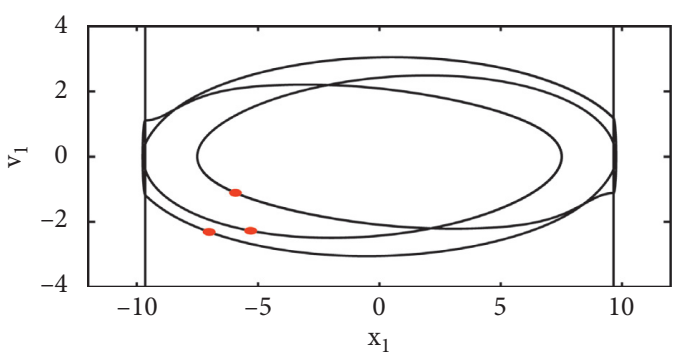

(c)

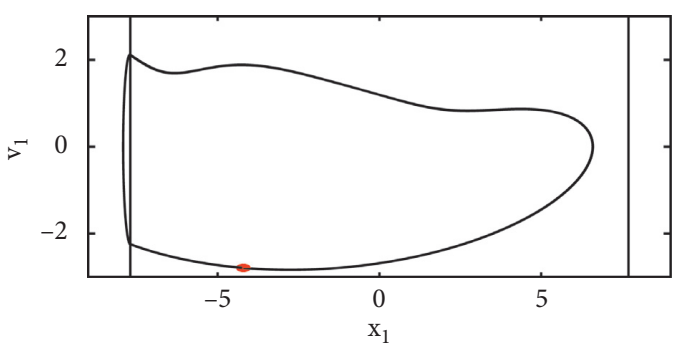

(e)

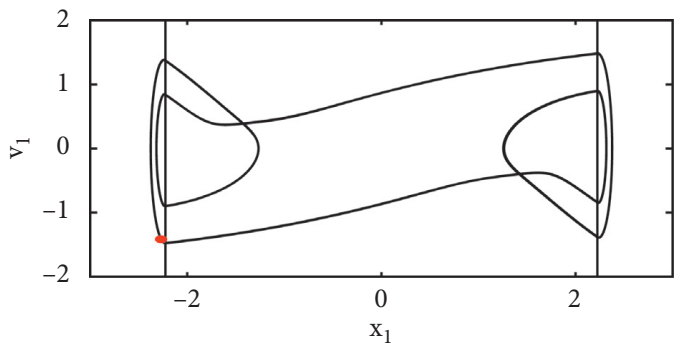

(g)

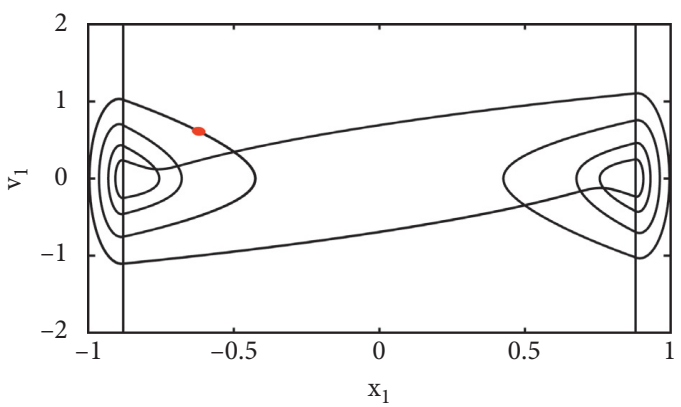

(i)

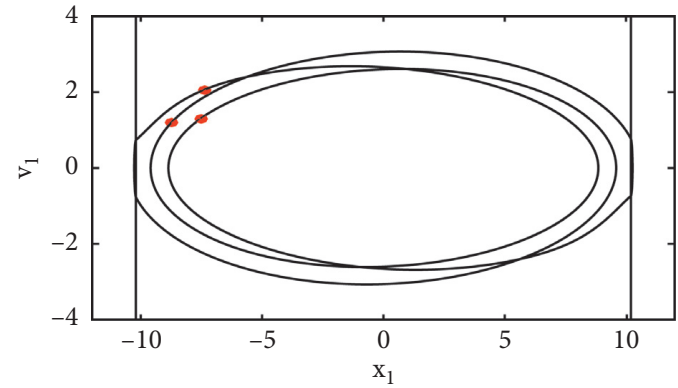

(b)

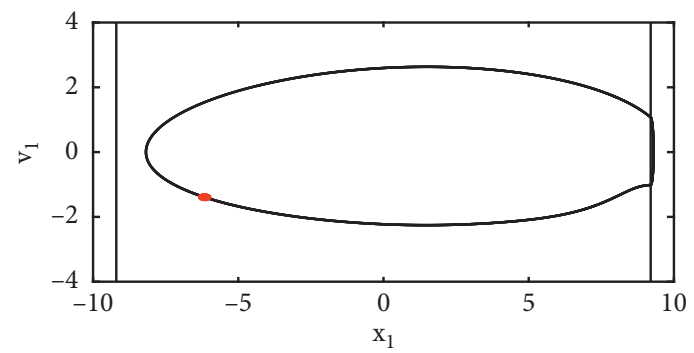

(d)

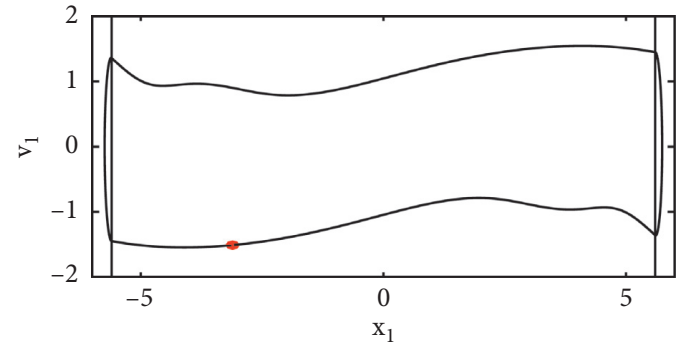

(f)

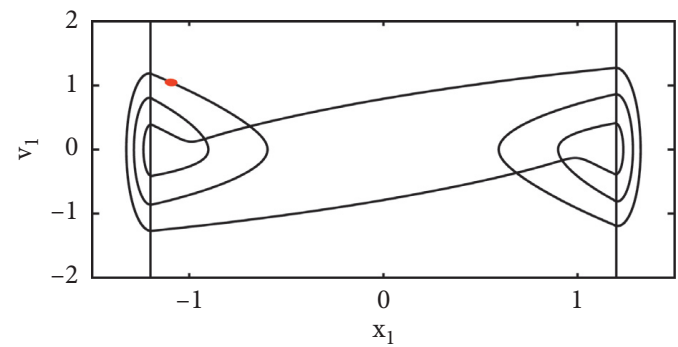

(h)

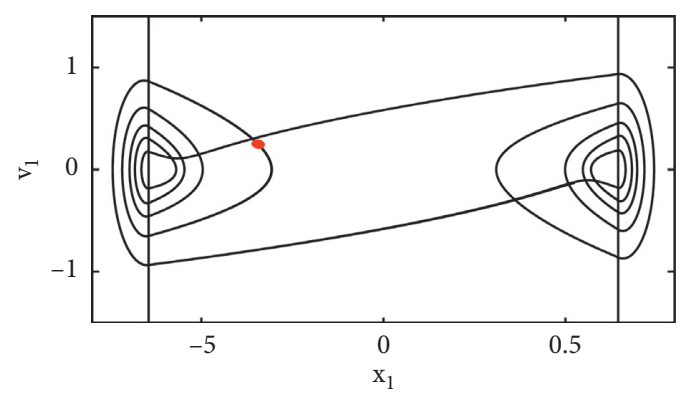

(j)

FIgURE 10: The phase diagrams in (a)-(j) correspond to the periodic motions of regions (j)-(s) in Figure 8, respectively. Poincare sections are marked by red dots on the phase planes. 
codimension-two grazing points. It can be seen from Figure 6 that the number of points of codimension-two grazing bifurcation increases with the decrease of frequency $\omega$.

When $n=1$, taking $\mu_{k}=0.25, \mu_{m}=0.25, \zeta=0.2$, a codimension-two grazing bifurcation point corresponds to $\omega=0.3096, d=10.88$, which satisfies the existence condition of grazing bifurcation. The grazing periodic trajectory is shown in Figure 7.

In order to further study the dynamic behavior of the system in the region near a codimension-two grazing bifurcation point, firstly fixed $\mu_{k}=0.25, \mu_{m}=0.25, \zeta=0.2$, $\mu_{k 0}=100$, and then $\omega=0.3096, d=10.88$ corresponds to the codimension-two grazing bifurcation point. Changing the values of the parameters $\omega, d$ in the vicinity of $\omega=0.3096, d=10.88$, different types of periodic-impact motions can be obtained by selecting a large number of parameters for numerical simulation. And finally, according to the dynamic behavior of the system, the parameter plane is divided into several regions. The unfolding diagram near the codimension-two grazing bifurcation point is drawn in Figure 8, where the dashed line in the diagram represents the grazing curve.

Selecting parameters in each area of Figure 8 for numerical simulation, a series of phase diagrams can be obtained. Fixing $\omega=0.32$, phase portraits obtained by perturbing $d$ are shown in Figures 9(a)-9(i). When we decrease $d$, the phase portraits corresponding to the regions (a)-(i) in Figure 8 are the $(1,1,3),(0,2,2),(0,1,1),(1,0,1)$, $(1,1,1),(2,2,1),(3,3,1),(4,4,1),(5,5,1)$ orbits, respectively.

For fixed $\omega=0.3$, phase portraits obtained by perturbing the parameter are shown in Figures $10(\mathrm{a})-10(\mathrm{j})$ corresponding to $(\mathrm{j})-(\mathrm{s})$ in Figure 8 , respectively. When we decrease $d$, the phase portraits corresponding to the regions $(\mathrm{j})-(\mathrm{s})$ in Figure 8 are the $(0,0,1),(1,1,3),(2,2,3),(0,1,1)$, $(1,0,1),(1,1,1),(2,2,1),(3,3,1),(4,4,1),(5,5,1)$ orbits, respectively.

\section{Conclusions}

For a two-degree-of-freedom system with symmetric elastic constraints, the codimension-one bifurcation of the system is discussed in detail by using the continuation method, and grazing bifurcation, saddle-node bifurcation, and perioddoubling bifurcation are obtained. As far as we knew, there are little research on the codimension-two grazing bifurcations of the system with the symmetric elastic constraints. Then, based on the traditional method of discontinuity mapping under the single constraint, we extend it to the impact oscillator with two-sided elastic constraints, and the specific mathematical expressions of zero-time-discontinuity mapping of the symmetric elastic constraint system are deduced after a complicated calculation. By combining the zero-time-discontinuity mappings and local smooth Poincare maps, the two composite mappings $\mathbf{P}_{1}, \mathbf{P}_{2}$ are obtained. According to the composite mappings, the criteria of codimension-two grazing bifurcation point under four different conditions are given out. The grazing curve and codimension-two grazing bifurcation points are shown by numerical simulation. And a series of complex dynamic behaviors in the vicinity of the codimension-two grazing bifurcation point are also presented, which reveal the rich dynamic behaviors of the impact system with two-sided elastic constraints.

\section{Data Availability}

The simulation data used to support the findings of this study are included within the article.

\section{Conflicts of Interest}

The authors declare that there are no conflicts of interest regarding the publication of this paper.

\section{Acknowledgments}

This work was supported by the National Natural Science Foundation of China (nos. 11872154 and 11372077).

\section{References}

[1] A. Colombo, M. Di Bernardo, S. J. Hogan, and M. R. Jeffrey, "Bifurcations of piecewise smooth flows: perspectives, methodologies and open problems," Physica D: Nonlinear Phenomena, vol. 241, no. 22, pp. 1845-1860, 2012.

[2] F. Dercole, A. Gragnani, and S. Rinaldi, "Bifurcation analysis of piecewise smooth ecological models," Theoretical Population Biology, vol. 72, no. 2, pp. 197-213, 2007.

[3] M. di Bernardo, A. Nordmark, and G. Olivar, "Discontinuityinduced bifurcations of equilibria in piecewise-smooth and impacting dynamical systems," Physica D: Nonlinear Phenomena, vol. 237, no. 1, pp. 119-136, 2008.

[4] M. di Bernardo, C. J. Budd, and A. R. Champneys, "Piecewisesmooth dynamical systems: theory and applications," Applied Mathematical Sciences, vol. 163, 2008.

[5] Y. Liu, M. Wiercigroch, E. Pavlovskaia, and H. Yu, "Modelling of a vibro-impact capsule system," International Journal of Mechanical Sciences, vol. 66, pp. 2-11, 2013.

[6] F. Angulo, G. Olivar, G. A. Osorio, C. M. Escobar, J. D. Ferreira, and J. M. Redondo, "Bifurcations of nonsmooth systems," Communications in Nonlinear Science and Numerical Simulation, vol. 17, no. 12, pp. 4683-4689, 2012.

[7] Y. Zhang and Q. Bi, "Non-smooth bifurcations in a doublescroll circuit with periodic excitation," Chinese Physics B, vol. 20, no. 1, pp. 1-9, 2011.

[8] G. Li, S. Wu, H. Wang et al., "Global behavior of a simplified model for the micro-vibration molding machine in parameter-state space," Mechanism and Machine Theory, vol. 154, pp. 1-19, 2020.

[9] J. Fan, J. Cao, S. Chen et al., "Discontinuous dynamic analysis of a class of three degrees of freedom mechanical oscillatory systems with dry friction and one-sided rigid impact," Mechanism and Machine Theory, vol. 151, pp. 1-30, 2020.

[10] J. Cao and J. Fan, "Discontinuous dynamical behaviors in a 2DOF friction collision system with asymmetric damping," Chaos, Solitons and Fractals, vol. 142, pp. 1-20, 2021.

[11] K. Witkowski, G. Kudra, G. Wasilewski et al., "Modelling and experimental validation of 1-degree-of-freedom impacting oscillator," Proceedings of the Institution of Mechanical Engineers Part I Journal of Systems and Control Engineering, vol. 233, no. 4, pp. 1-13, 2019. 
[12] M. Gao, J. Fan, and C. Li, "Analysis of discontinuous dynamics of a 2-DOF system with constrained spring cushions," International Journal of Non-linear Mechanics, vol. 128, pp. 1-16, 2021.

[13] G. Li and W. Ding, "Global behavior of a vibro-impact system with asymmetric clearances," Journal of Sound and Vibration, vol. 423, no. 4-5, pp. 180-194, 2018.

[14] G. Li, S. Wu, H. Wang et al., "Global dynamics of a nonsmooth system with elastic and rigid impacts and dry frictionScienceDirect," Communications in Nonlinear Science and Numerical Simulation, vol. 95, pp. 1-25, 2020.

[15] G. Li, W. Ding, and S. Wu, "Global behavior of a vibro-impact system with multiple nonsmooth mechanical factors," Journal of Computational and Nonlinear Dynamics, vol. 12, no. 6, pp. 1-43, 2017.

[16] A. C. J. Luo, "On the symmetry of solutions in non-smooth dynamical systems with two constraints," Journal of Sound and Vibration, vol. 273, no. 4-5, pp. 1118-1126, 2004.

[17] Y. Yue and J. Xie, "Symmetry and bifurcations of a twodegree-of-freedom vibro-impact system," Journal of Sound and Vibration, vol. 314, no. 1-2, pp. 228-245, 2008.

[18] S. W. Shaw and P. J. Holmes, "A periodically forced impact oscillator with large dissipation," Journal of Applied Mechanics, vol. 50, no. 4a, pp. 849-857, 1983.

[19] H. Jiang and M. Wiercigroch, "Geometrical insight into nonsmooth bifurcations of a soft impact oscillator," IMA Journal of Applied Mathematics, vol. 81, no. 4, pp. 662-678, 2016.

[20] J. Ing, E. Pavlovskaia, M. Wiercigroch, and S. Banerjee, "Bifurcation analysis of an impact oscillator with a one-sided elastic constraint near grazing," Physica D: Nonlinear Phenomena, vol. 239, no. 6, pp. 312-321, 2010.

[21] W. Du, S. Zhang, and S. Yin, "An intermittent chaos control method for a class of symmetric impact systems," Applied Mathematics and Mechanics, vol. 39, no. 10, pp. 1149-1158, 2018.

[22] H. Gritli, "Robust master-slave synchronization of chaos in a one-sided 1-DoF impact mechanical oscillator subject to parametric uncertainties and disturbances," Mechanism and Machine Theory, vol. 142, pp. 1-22, 2019.

[23] F. Turki, H. Gritli, and S. Belghith, "An LMI-based design of a robust state-feedback control for the master-slave tracking of an impact mechanical oscillator with double-side rigid constraints and subject to bounded-parametric uncertainty," Communications in Nonlinear Science and Numerical Simulation, vol. 82, pp. 1-26, 2020.

[24] Y. Shen, S. Yin, G. Wen et al., "Feedback control of grazinginduced chaos in the single-degree-of-freedom impact oscillator," Journal of Computational and Nonlinear Dynamics, vol. 13, pp. 1-9, 2018.

[25] S. Yin, G. Wen, and X. Xu, "Suppression of grazing-induced instability in single degree-of-freedom impact oscillators," Applied Mathematics and Mechanics, vol. 40, no. 1, pp. 97$110,2019$.

[26] H. Gritli and S. Belghith, "Diversity in the nonlinear dynamic behavior of a one-degree-of-freedom impact mechanical oscillator under OGY-based state-feedback control law: order, chaos and exhibition of the border-collision bifurcation," Mechanism and Machine Theory, vol. 124, pp. 1-41, 2018.

[27] H. Gritli and S. Belghith, "Walking dynamics of the passive compass-gait model under OGY-based state-feedback control: analysis of local bifurcations via the hybrid Poincaré map," Chaos, Solitons \& Fractals, vol. 98, pp. 72-87, 2017.
[28] H. Gritli and S. Belghith, "Walking dynamics of the passive compass-gait model under OGY-based state-feedback control: rise of the Neimark-Sacker bifurcation," Chaos, Solitons \& Fractals, vol. 110, pp. 158-168, 2018.

[29] H. Gritli and S. Belghith, "Walking dynamics of the passive compass-gait model under OGY-based control: emergence of bifurcations and chaos," Communications in Nonlinear Science and Numerical Simulation, vol. 47, pp. 308-327, 2017.

[30] J. P. Chávez, P. Brzeski, and P. Perlikowski, "Bifurcation analysis of non-linear oscillators interacting via soft impacts," International Journal of Non-linear Mechanics, vol. 92, pp. 76-83, 2017.

[31] A. B. Nordmark, "Non-periodic motion caused by grazing incidence in an impact oscillator," Journal of Sound and Vibration, vol. 145, no. 2, pp. 279-297, 1991.

[32] A. B. Nordmark, "Universal limit mapping in grazing bifurcations," Physical Review E, vol. 55, no. 1, pp. 266-270, 1997.

[33] W. Chin, E. Ott, H. E. Nusse, and C. Grebogi, "Grazing bifurcations in impact oscillators," Physical Review E, vol. 50, no. 6, pp. 4427-4444, 1994.

[34] H. Dankowicz and A. B. Nordmark, "On the origin and bifurcations of stick-slip oscillations," Physica D:Nonlinear Phenomena, vol. 136, no. 3-4, pp. 280-302, 2000.

[35] M. D. Bernardo, C. J. Budd, and A. R. Champneys, "Normal form maps for grazing bifurcations in $\mathrm{n}$-dimensional piecewise-smooth dynamical systems," Physica D:Nonlinear Phenomena, vol. 160, no. 3-4, pp. 222-254, 2001.

[36] J. Xu, Q. Li, and N. Wang, "Existence and stability of the grazing periodic trajectory in a two-degree-of-freedom vibroimpact system," Applied Mathematics and Computation, vol. 217, no. 12, pp. 5537-5546, 2011.

[37] S. Yin, Y. Shen, G. Wen, and H. Xu, "Analytical determination for degenerate grazing bifurcation points in the single-degreeof-freedom impact oscillator," Nonlinear Dynamics, vol. 90, no. 1, pp. 443-456, 2017.

[38] S. Yin, J. Ji, S. Deng et al., "Neimark-sacker bifurcations near degenerate grazing point in a two degree-of-freedom impact oscillator," Journal of Computational and Nonlinear Dynamics, vol. 13, no. 11, pp. 1-8, 2018.

[39] S. Yin, J. Ji, S. Deng et al., "Degenerate grazing bifurcations in a three-degree-of-freedom impact oscillator," Nonlinear Dynamics, vol. 97, no. 2, pp. 525-539, 2019.

[40] S. Yin, G. Wen, J. Ji et al., "Novel two-parameter dynamics of impact oscillators near degenerate grazing points," International Journal of Non-linear Mechanics, vol. 120, pp. 1-11, 2020.

[41] S. Yin, J. Ji, and G. Wen, "Complex near-grazing dynamics in impact oscillators," International Journal of Mechanical Sciences, vol. 156, pp. 106-122, 2019.

[42] S. Yin, G. Wen, H. Xu, and X. Wu, "Higher order zero time discontinuity mapping for analysis of degenerate grazing bifurcations of impacting oscillators," Journal of Sound and Vibration, vol. 437, pp. 209-222, 2018.

[43] P. Kowalczyk, M. Di Bernardo, A. R. Champneys et al., "Two-parameter discontinuity-induced bifurcations of limit cycles: classification and open problems," International Journal of Bifurcation and Chaos, vol. 16, no. 03, pp. 601-629, 2006.

[44] H. Dankowicz and X. Zhao, "Local analysis of co-dimensionone and co-dimension-two grazing bifurcations in impact microactuators," Physica D:Nonlinear Phenomena, vol. 202, no. 3-4, pp. 238-257, 2005. 
[45] P. Thota, X. Zhao, and H. Dankowicz, "Co-dimension-Two grazing bifurcations in single-degree-of-freedom impact oscillators," Journal of Computational and Nonlinear Dynamics, vol. 1, no. 4, pp. 328-335, 2006.

[46] J. Xu, P. Chen, and Q. Li, "Theoretical analysis of co-dimension-two grazing bifurcations in single-degree-of-freedom impact oscillator with symmetrical constrains," Nonlinear Dynamics, vol. 82, no. 4, pp. 1641-1657, 2015. 